\title{
Tradable Network Permits: \\ A New Scheme for the Most Efficient Use of Network Capacity
}

\author{
Takashi Akamatsu \\ Graduate School of Information Sciences, Tohoku University, Sendai, Miyagi,980-8579, Japan \\ E-mail : akamatsu@plan.civil.tohoku.ac.jp
}

\begin{abstract}
Akamatsu et al.(2006) proposed a new transportation demand management scheme called "tradable bottleneck permits" (TBP), and proved its efficiency properties for a single bottleneck model. This paper explores the properties of a TBP system for general networks. An equilibrium model is first constructed to describe the states under the TBP system. It is proved that equilibrium resource allocation is efficient in the sense that the total transportation cost in a network is minimized. Theoretical relationships between TBP and congestion pricing (CP) are also discussed. It is demonstrated that TBP has definite advantages over $\mathrm{CP}$ when demand information is not perfect, whereas both TBP and $\mathrm{CP}$ are equivalent for the perfect information case. Finally, it is shown that the "self-financing principle" also holds for the TBP system.
\end{abstract}

Key Words : bottleneck congestion, dynamic traffic assignment, time-space network, tradable permit, ITS

Recent advances in information and communication technology (ICT) have led to rapid changes in the "virtual" world represented by the Internet. The increasing capabilities and decreasing cost of ICT is now becoming the impetus for changing the "real" world. The effects of ICT on transportation systems are no exception. The broadly defined "Intelligent Transportation Systems (ITS)" that exploits ICT has a large potential for dramatically improving efficiency of road transportation systems if the systems are implemented together with appropriate transportation demand management (TDM) schemes.

As an example of such futuristic TDM schemes making the most of ICT/ITS, Akamatsu, Sato and Nguyen (2006), and Akamatsu (2007a) proposed the "tradable bottleneck permit system." Their proposed scheme is designed for resolving the problem of congestion during the morning rush hour at a single bottleneck, and consists of the following two parts:

a) the road manager issues a right that allows a permit holder to pass through the bottleneck at a pre-specified time period ("bottleneck permits"),

b) a new trading market is established for bottleneck permits differentiated by a pre-specified time

Note here that both parts $a$ ) and $b$ ) of this scheme are feasible for implementation from a technical point of view, even at the present time. The system for handling part $a$ ) may be constructed as an application of the dedicated short range communication (DSRC) system that is used in the current electric toll collection (ETC) system; the trading markets in part $b$ ) also can be realized inexpensively by using Internet auction markets. It is, therefore, reasonable to assume that implementing this scheme will become technically easier when we take into account the future advances of ICT/ITS. 
Part $a$ ) of this scheme is almost the same as the concept of "advance highway booking" (reservations or quotas)that has been previously proposed by several authors (e.g., Akahane and Kuwahara (1996), Wong (1997), Teodorovic and Edara (2005)). Under this scheme, the arrival flow rate at a bottleneck at any time period is, from the definition of the scheme, equal to the number of permits issued for that time period. This implies that we can completely eliminate the occurrence of queuing congestion by setting the number of permits issued per unit time to be less than or equal to the bottleneck capacity. However, there may be cases in which road users cannot choose their desired time for using the road if the permits are assigned according to some unrefined rule (e.g., a simple "quota" scheme). Such an infringement on freedom of choice necessarily causes economic losses and should not be socially acceptable. In order to circumvent this problem that arises in employing only part $a$ ) of the scheme, we need to add an appropriate mechanism in which each user can choose his or her desired permit. It is part $b$ ) of the scheme that gives the foundation for this "choice mechanism” by a market system for buying and selling permits.

With the complementary properties of parts $a$ ) and $b$ ) in the combined scheme above, we can expect that this is the most efficient scheme of using the limited resource of road capacity. Indeed, for a departure-time choice equilibrium problem with a single bottleneck a la Vickrey (1969), Akamatsu (2007a) showed that the proposed system has the following desirable properties: (1) Comparing equilibrium states with and without the proposed system, we can achieve Pareto improvement for both the road manager and all road users, (2) the equilibrium with the proposed system achieves the most efficient (i.e., Pareto optimal) resource allocation, (3) the "self-financing principle" holds for the equilibrium with the proposed system-that is, the total revenue (market value) of selling the permits is equal to the investment cost required for increasing the bottleneck capacity to a socially optimal level.

These properties of the tradable permit system are proved only for a road with a single bottleneck. Specifically, the proof is based on the isomorphism between the commuters' departuretime equilibrium in a single bottleneck model and an equilibrium model of an urban residential location (see, for example, Fujita (1989)). Since such isomorphism cannot be extended to a case with multiple bottlenecks, the properties of the tradable permit system for general networks are largely unknown. Furthermore, we should note here that there are no studies dealing with tradable permits in the transportation field, other than Verhoef, Nijkamp and Rietveld (1997), Akamatsu et al. (2006) and Akamatsu (2007a). Although Verhoef et al. (1997) give an excellent discussion on the possibilities of tradable permits in road transportation, they do not mention time-dependent tradable permits for eliminating bottleneck congestion. Thus, whether or not the above desirable properties hold for general networks is a problem yet to be studied.

The purpose of this paper is to explore some of the properties of a system of tradable bottleneck permits for general networks (we call this a system of "tradable network permits.”). Specifically, after defining the system of a tradable network permit, we present a mathematical model that describes the equilibrium that arises under the tradable permit system. We then prove that the equilibrium resource allocation under the system is efficient in the sense that the total transportation cost in a network is minimized: formulating a dynamic system optimal assignment, we show that the equilibrium assignment coincides with the optimal assignment; we also show that the feasibility of the optimal assignment can be easily confirmed by constructing a "time-space network." We further show the theoretical relationship between the tradable permit system and congestion pricing: we demonstrate the definite advantages of the tradable permit system over 
congestion pricing when the demand information is not perfect, whereas they are equivalent for the perfect information case. Finally, we prove that the "self-financing principle" holds not only for the single bottleneck case but also for the general network case.

The organization of this paper is as follows. In Section 1, we outline the framework of the tradable permits system analyzed in this paper. In Section 2, we present a model of the equilibrium under the tradable network permit system. In Section 3, we analyze the efficiency of the equilibrium allocation. In Section 4, we discuss the theoretical relationship between the tradable permit system and congestion pricing. Section 5 shows that the self-financing principle holds for tradable network permits. Finally, Section 6 concludes the paper.

\section{A System of Tradable Bottleneck Permits in Transportation Networks}

\subsection{Networks}

In this paper, we consider dynamic traffic flows on a general network $G$ (i.e., a transportation network with general topology). The network consists of a set $N$ of nodes, and a set $L$ of directed links. The node set $N$ includes an origin node from which users start their trips, and a destination node at which users terminate their trips. To avoid notational complexity and to outline essential aspects of the theory, we deal with networks with a single origin-destination (OD) pair. Each element of $N$ (i.e., each node) is identified by a sequential natural number $i$, and each element of $L$ (i.e., each link) is denoted by a pair $(i, j)$ of the upstream node $i$ and the downstream node $j$. The time interval $I=[0, T]$ for which we assign the dynamic traffic flow is fixed. We assume that the travel demand $Q$ that makes trips for the time interval $I$ is a given constant.

We also assume, without any loss of generality, that each link in a network consists of a free flow segment and a single bottleneck segment. The travel time to pass through the free flow segment of link $(i, j)$ is a constant $t_{i j}$ (i.e., $t_{i j}$ is independent of time and flow). The bottleneck of each link is represented by a point queue model with constant capacity $\mu_{i j}$. Note that this modeling approach can deal with any number of bottlenecks in a road segment. When we wish to consider a segment without a bottleneck, we set the capacity as infinity; when we consider a segment with multiple bottlenecks, we just set up multiple links corresponding to each bottleneck.

\subsection{Agents}

The model presented in this paper has two types of agents: a road network manager and road network users. The road manager aims to restrain traffic congestion on the network and minimize the "social transportation cost." To achieve this, the manager regulates the traffic flow rates entering into each bottleneck in the network by using "time-dependent bottleneck permits." The precise definition and setup of the bottleneck permit system are described in Subsection $\mathbf{1 . 3}$ below.

Each user makes a single trip (for the time interval $I$ ) from an origin (e.g., residential zone) to a destination (e.g., central business district (CBD)) in the network. The user chooses a destination arrival time and a path between the origin and the destination so as to minimize his or her disutility (or "generalized transportation cost"). The detailed definition of disutility is mentioned in Section 2. Under the bottleneck permits system, each user must purchase a set of permits corresponding to a set of links included in the user's chosen path. This implies that choosing a destination arrival time and a path directly links to purchasing time-dependent bottleneck permits in the "permits markets." 
More detailed explanations on the purchase scheme and the permit markets are given in Subsections 1.3 and 1.4.

\subsection{Bottleneck Permits}

"Time-dependent bottleneck permits" is a right that allows the permit holder to pass through a pre-specified bottleneck at a pre-specified time. In this paper, we assume that the road manager can issue time-dependent bottleneck permits for all bottlenecks (i.e., links) in the network. This implies that the traffic flow entering into link $(i, j)$ at time $t$ consists of only users who have a "time $t$ permit for link $(i, j)$," and users without this permit cannot pass through this link at this time.

Throughout this paper, we assume that the number of permits issued for each link for each unit time is equal to or less than the traffic capacity of each link in the network. This means that queuing congestion never occurs in the network under this permits-issue scheme. This may be easily seen from this explanation of permits: the inflow rate of each link is equal to (or less than) the number of permits issued, and hence the inflow rate cannot exceed the traffic capacity of each link, which implies that queuing congestion at each link can never occur.

\subsection{Trading Markets of Bottleneck Permits}

For assigning the bottleneck permits to users, we can consider two representative schemes: "market selling scheme" and "free distribution scheme" (Akamatsu (2007a)). In the "market selling" scheme, the road manager sells all the bottleneck permits to users in "bottleneck permits markets.” All sales from selling the permits result in revenue for the road manager in this scheme. In the "free distribution" scheme, the road manager distributes all the permits to users for free according to methods that consider the equity among users. In this scheme, the time designated by the permits assigned for each user does not necessarily match one's own desired arrival time. For that case, users can mutually trade a wide variety of permits in the bottleneck permits markets: each user can buy the necessary permits while selling the unnecessary permits. As a result of such trading activities, permits are re-distributed among users through a system of prices emerging in the market. Thus, all income transfers take place only among the users in this scheme.

This paper deals with only the former scheme ("market selling scheme") to make the exposition as simple as possible. The permits issued for each link (bottleneck) are put on sale by the road manager. There are as many markets for trading permits as there are links, and each market is dedicated for trading the permits for each link. The permits for each link are further distinguished by a specified time allowable to use the link. Under the bottleneck permits system, each user who would like to use a path must have a set of permits corresponding to a set of links included in the path before making a trip. To fulfill this requirement, each user is assumed to purchase the needed set of permits in the trading markets. The price of each permit is determined by an auction system, which implies that the price is adjusted so as to clear the excess demand for each type of permit. We also assume that the markets are perfectly competitive; that is, neither a monopoly nor oligopoly occurs.

\section{Equilibrium under a System of Tradable Network Permits}

This section provides a model of equilibrium that takes place after introducing the tradable network permits system. We first describe the conditions that should be satisfied by several 
dynamic transportation cost variables, and a model of users' behaviors for choosing permits is shown. We then formulate the equilibrium under the tradable permits system.

\subsection{Dynamic Travel Costs in General Networks}

The transportation cost for a single trip of a network user consists of the following three types of costs: a) “schedule cost,” b) “travel cost,”c) “permit purchase cost.”

a) The "schedule cost" for a user is the cost due to the difference between the user's desired arrival time and the actual arrival time $t$. The desired arrival time is assumed to be the same for all users and is equal to $s$. The schedule cost is represented by the function $w(t)$ of destination arrival time $t$, which is common to all users, and is assumed to be a strictly convex function with a minimum at $s=t$, following previous studies on the departure time equilibrium (e.g., Smith (1984), Daganzo (1985), Kuwahara (1990)).

b) The "travel cost" is the monetary equivalent of the travel time needed for a trip from the origin to the destination. The travel times are different among paths. The travel time of a path between the origin-destination pair is defined as the sum of travel times of the links included in the path. Note that the travel time of each link $(i, j)$ is a constant $t_{i j}$ at equilibrium under the permits system, in which no queuing occurs. Hence, at equilibrium, the travel time $T_{r}$ of path $r$ between the origin-destination pair arrival time is also constant:

$$
T_{r}=\sum_{i j \in L} t_{i j} \delta_{i j, r(o, d)}
$$

where $\delta_{i j, r(o, d)}$ is a typical element of the path-link incidence matrix for node pair $(o, d)$ : it is 1 if link $(i, j)$ is on path $r$ connecting OD pair $(o, d)$; otherwise, it is zero.

c) The "permit purchase cost" is the total payment for purchasing a set of link permits required for going through a path from the origin to the destination. To put it another way, the permit purchase cost of a user is defined as the sum of permit prices of the links included in the path used. This cost varies depending on what path is taken and at what time because the permits for each link are further differentiated by the specified time and each permit is priced depending on the time and the link.

To see this cost more precisely, consider a user who uses path $r$ and arrives at the destination at time $t$. Suppose here that he or she uses a path $r$ that contains link $(i, j)$ and $T_{i j, r}$ is the travel time required for arriving at the destination from node $i$ :

$$
T_{i j, r}=\sum_{k \ell \in L} t_{k \ell} \delta_{k \ell, r(i, d)}
$$

where $\delta_{k l, r(i, d)}$ is a typical element of the path-link incidence matrix for node-pair $(i, d)$ : it is 1 if link $(k, l)$ is on path $r$ from node $i$ to the destination $d$; otherwise, it is zero. Then, the user should enter into the link at time $t-T_{i j, r}$, which implies that this user has to obtain the time $t-T_{i j, r}$ permit for link $(i, j)$, whose price is $p_{i j}\left(t-T_{i j}, r\right)$. It follows from this that the permit purchase cost (i.e., the total payment for purchasing the set of link permits required for going through path $r$ and arriving at the destination at time $t$ ) is given by

$$
P_{r}(t)=\sum_{i j \in L} p_{i j}\left(t-T_{i j, r}\right) \delta_{i j, r(o, d)}
$$


We call the sum of the travel cost and permit purchase cost as the path transportation cost; that is, the transportation cost $C_{r}(t)$ of path $r$ for a user arriving at the destination at time $t$ is given by

$$
C_{r}(t)=P_{r}(t)+\alpha T_{r}
$$

where $\alpha$ is a coefficient that converts travel time to the monetary equivalent.

\subsection{Users' Behaviors}

Each user chooses an arrival time $t$ at the destination and a path $r$ between the origin and the destination so as to minimize the generalized transportation cost, defined as the sum of the schedule cost and transportation cost. That is, the user solves the following problem:

$$
\min _{t, r} . w(t)+C_{r}(t)
$$

This optimization problem can be solved by "backward induction.” That is, the optimal choice pair $\left(t^{*}, r^{*}\right)$ can be obtained by solving a two-stage (hierarchical) choice problem, in which one chooses separately the destination arrival time $t$ (the upper-level choice) and the optimal path $r$ (the lowerlevel choice). More specifically, we first solve the lower-level problem of the path choice for a given arrival time $t$; we then obtain the optimal route choice $r^{*}(t)$ and the optimal value $\pi(t)$ conditional on arrival time $t$ :

$$
\begin{array}{r}
\pi(t)=\min _{r} \cdot C_{r}(t) \\
r^{*}(t)=\arg \cdot \min _{r} \cdot C_{r}(t)
\end{array}
$$

By using this optimal choice function, we can reduce the upper-level problem of the arrival time choice to

$$
\begin{array}{r}
\min _{t} \cdot w(t)+\pi(t) \\
t^{*}=\arg \cdot \min _{t} \cdot w(t)+\pi(t)
\end{array}
$$

\subsection{Equilibrium Conditions}

For the settings above, we construct a model that describes the equilibrium that arises in the presence of the proposed system. At equilibrium, the following six conditions as well as the definitional equations (1)-(4) for time-dependent cost variables should hold.

\section{1) Flow conservation for path flows and OD flows:}

OD flow rate $q(t)$ for a user arriving at the destination at time $t$ should be the sum of the path flow rates:

$$
\sum_{r \in R} f_{r}(t)=q(t)
$$$$
\forall t \in I
$$

where $f_{r}(t)$ is the flow rate of path $r$ arriving at the destination at time $t ; R$ is the set of paths connecting the origin-destination pair.

\section{2) Equilibrium conditions for the path choice:}

As mentioned in Subsection 2.2, the user's optimal choice problem can be solved by "backward induction." By first obtaining the solution of the path choice problem as a function of arrival time $t$, we then solve the arrival time choice problem. To obtain the equilibrium condition for the first problem, consider a user arriving at the destination at time $t$. At equilibrium, no user can improve his or her own cost by changing the path choice unilaterally (i.e., no user has the incentive 
to deviate from his or her strategy). That is, transportation cost $C_{r}(t)$ of path $r$ (from the origin to the destination) that is chosen at equilibrium is equal to the minimum path travel cost $\pi(t)$, and the costs of other (unused) paths are greater than $\pi(t)$ :

$$
\left\{\begin{array}{l}
\pi(t)=C_{r}(t) \text { if } f_{r}(t)>0 \\
\pi(t) \leq C_{r}(t) \text { if } f_{r}(t)=0
\end{array} \quad \forall t \in I, \forall r \in R\right.
$$

\section{3) Flow conservation for OD flow rates and OD travel demands:}

All OD travel demands $Q$ have to be assigned to each time point (in terms of the arrival time at the destination) in the interval $[0, T]$; that is, the time-dependent OD flow rates $\{q(t)\}$ should satisfy

$$
\int_{0}^{T} q(u) d u=Q
$$

\section{4) Equilibrium conditions for destination arrival time choice:}

At equilibrium, no one can improve his or her own generalized transportation cost by changing the destination arrival time unilaterally. It follows from the path choice equilibrium condition in 2) that the generalized transportation cost for a user arriving at time $t$ is $w(t)+\pi(t)$, where $w(t)$ is the schedule cost for a user arriving at the destination at time $t$. Therefore, the equilibrium condition for the user's arrival time choice can be expressed as

$$
\left\{\begin{array}{ll}
\rho=\pi(t)+w(t) & \text { if } q(t)>0 \\
\rho \leq \pi(t)+w(t) & \text { if } q(t)=0
\end{array} \quad \forall t \in I\right.
$$

where $\rho$ represents the minimum (equilibrium) generalized transportation cost.

\section{5) Demand-supply equilibrium (market clearing) conditions in each link permit market:}

As mentioned in Section 1, the permits for each link are distinguished by a specified time at which each permit makes it allowable to use the link. Let $p_{i j}(t)$ denote the price of the permit for link $(i, j)$ with a specified allowance time $t$. Since the trading markets are assumed to be perfectly competitive (i.e., neither a monopoly nor oligopoly occurs), the price $p_{i j}(t)$ of each permit type is adjusted to clear the excess demand for each type of permit. More precisely, at equilibrium, if the price of a certain type of permit is positive, the quantities supplied and the quantities demanded for the permit are equal; for the permit whose supply quantity exceeds the quantity demanded, the price is zero. Note here that, for each link $(i, j)$ and each allowance time $t$, the demand of the time $t$ permit of the link is equal to the inflow rate $y_{i j}(t)$. On the other hand, the maximum supply (upper bound) of the time $t$ permit of link $(i, j)$ is given by the link capacity $\mu_{i j}$. Therefore, the demandsupply equilibrium condition for the permits market is represented as

$$
\left\{\begin{array}{ll}
y_{i j}(t)=\mu_{i j} & \text { if } p_{i j}(t)>0 \\
y_{i j}(t) \leq \mu_{i j} & \text { if } p_{i j}(t)=0
\end{array} \quad \forall t \in I, \forall i j \in L\right.
$$

\section{6) Flow conservation for link flows and path flows:}

The inflow rate on each link at each time point should be consistent with the timedependent path flows. A user going through path $r$ and entering into link $(i, j)$ at time $t$ arrives at the destination at time $t+T_{i j, r}$. Therefore, the inflow rate $y_{i j}(t)$ entering into link $(i, j)$ at time $t$ is the sum of the flows on all paths going through that link and arriving at the destination at $t+T_{i j, r}$ :

$$
y_{i j}(t)=\sum_{r \in R} f_{r}\left(t+T_{i j, r}\right) \delta_{i j, r(o, d)} \quad \forall t \in I, \forall i j \in L
$$




\subsection{Arc-Node Formulation}

The path-based formulation presented above is convenient for presenting the user's route choice behavior in a straightforward manner; however, this formulation is not necessarily convenient for analyzing the demand-supply equilibrium condition (12) at each link due to the complexity of the relationship (13) between time-dependent path flows and link flows. In order to alleviate this complexity, we transform the model above into a formulation expressed in terms of link-node variables, which enables us to analyze the efficiency of the equilibrium, as is presented in later sections. The arc-node formulation of the model can be summarized by the following five conditions.

\section{1a) Flow conservation at each node:}

Conservation of the dynamic traffic flow in a network is represented as the equality of inflow and outflow at each node at each time point. To formalize this, let $y_{i j}(t)$ be the flow rate arriving at link $(i, j)$ at time $t$, and $z_{i j}(t)$ be the flow rate departing from link $(i, j)$ at time $t$. Then the flow conservation is represented as

$$
\sum_{k \in N O(i)} y_{i k}(t)-\sum_{k \in N I(i)} z_{k i}(t)=-q(t) \delta_{i d}, \quad \forall t \in I, \forall i \in N
$$

where $\delta_{\text {id }}$ is Kronecker's delta (i.e., 1 if $i=d$, zero otherwise ); $N O(i)$ is a set of downstream nodes of the links incident from node $i$; NI (i) is a set of upstream nodes of the links incident to node $i$.

\section{1b) First-In-First-Out conditions on each link :}

We assume that the dynamic traffic flow in our model should satisfy the First-In-First-Out (FIFO) property on each link (i.e., we assume that passing can be neglected). As shown in the literature (see, for example, Kuwahara and Akamatsu (1993), Akamatsu and Kuwahara (1994)), the FIFO condition for each link can be written as

$$
A_{i j}(t)=D_{i j}\left(t+t_{i j}(t)\right),
$$

where $A_{i j}(t)$ and $D_{i j}(t)$ are the cumulative numbers of vehicles entering into and leaving from link $(i, j)$ at time $t$, respectively. Using the flow rate variables, we can equivalently rewrite this as

$$
y_{i j}(t)=z_{i j}\left(t+t_{i j}(t)\right) \cdot\left(1+d t_{i j}(t) / d t\right),
$$

where $t_{i j}(t)$ is the travel time of link $(i, j)$ for a user entering into the link at time $t$. Note here that $t_{i j}(t)$ is a constant regardless of the arrival time when there is no queue. Hence, at equilibrium under the permits system (i.e., when there is no queue in the network), the FIFO condition (16) reduces to the following simpler representation:

$$
y_{i j}(t)=z_{i j}\left(t+t_{i j}\right) \quad \forall t \in I, \forall i j \in L
$$

\section{2) Equilibrium conditions for path choice:}

Consider a user arriving at node $i$ at time $t$. If the user chooses link $(i, j)$, the arrival time at node $j$ is $t+t_{i j}$. Hence, at equilibrium, link $(i, j)$ should be on the minimum path for a user arriving at node $j$ at time $t+t_{i j}$ if there exists a user entering into link $(i, j)$ at time $t$. Denoting $\pi_{i}(t)$ as the minimum path cost from the origin to node $i$ for a user arriving at the node at time $t$, we can represent this condition as

$$
\left\{\begin{array}{l}
\pi_{j}\left(t+t_{i j}\right)=c_{i j}(t)+\pi_{i}(t) \text { if } y_{i j}(t)>0 \\
\pi_{j}\left(t+t_{i j}\right) \leq c_{i j}(t)+\pi_{i}(t) \text { if } y_{i j}(t)=0
\end{array} \quad \forall t \in I, \forall i j \in L\right.
$$

where $c_{i j}(t)$ is the transportation cost for a user who enters into link $(i, j)$ at time $t$ : 


$$
c_{i j}(t) \equiv p_{i j}(t)+\alpha t_{i j}
$$

The condition (18) is equivalent to the minimum cost path choice condition (9) represented by the path variables since (18) can be derived by applying the dynamic programming principle to (9).

3) Flow conservation for OD flow rates and OD travel demands:

This is the same as the conditions in 3) in Subsection 2.3, that is,

$$
\int_{0}^{T} q(u) d u=Q
$$

\section{4) Equilibrium conditions for destination arrival time choice:}

Minimum path cost $\pi(t)$ defined in Subsection 2.3 is the same as $\pi_{d}(t)$ defined in (18) above. Hence, in a similar manner to the conditions in 4) in Subsection 2.3, the equilibrium conditions for the user's choice of destination arrival times is given by

$$
\left\{\begin{array}{l}
\rho=\pi_{d}(t)+w(t) \text { if } q(t)>0 \\
\rho \leq \pi_{d}(t)+w(t) \text { if } q(t)=0
\end{array}\right.
$$$$
\forall t \in I
$$

5) Demand-supply equilibrium (market clearing) conditions in each link permit market:

This is the same as the conditions in 3) in Subsection 2.3, that is,

$$
\left\{\begin{array}{ll}
y_{i j}(t)=\mu_{i j} & \text { if } p_{i j}(t)>0 \\
y_{i j}(t) \leq \mu_{i j} & \text { if } p_{i j}(t)=0
\end{array} \quad \forall t \in I, \forall i j \in L\right.
$$

\section{Efficiency of the Equilibrium with Tradable Network Permits}

\subsection{Dynamic System Optimal Assignment without Queuing}

In order to examine the efficiency of the equilibrium allocation patterns defined in (14)(22), consider the following optimization problem [P-1]:

$$
\begin{aligned}
& \min _{(\mathbf{q}, \mathbf{y}) \geq \mathbf{0}} F_{P}(\mathbf{q}, \mathbf{y}) \equiv \int_{0}^{T} q(t) w(t) d t+\alpha \sum_{i j \in L} \int_{0}^{T} y_{i j}(t) t_{i j} d t \\
& \text { subject to } \\
& \int_{0}^{T} q(u) d u=Q \\
& y_{i j}(t) \leq \mu_{i j} \\
& \forall t \in I, \forall i j \in L \\
& \sum_{k \in N O(i)} y_{i k}(t)-\sum_{k \in N I(i)} y_{k i}\left(t-t_{k i}\right)=-q(t) \delta_{i d}
\end{aligned}
$$

This is the problem of finding a dynamic traffic flow pattern that minimizes the total generalized transportation cost in the network, subject to the physical constraints of flows representing the network performance. Specifically, the first term of the objective function $F_{P}$ is the total schedule cost expensed by all users, and the second term is the monetary equivalent of the total travel time paid by all users. The first constraint (24) is conservation of the OD demand, the second constraint (25) is the traffic capacity constraints on each link. The final constraint (26) is the conservation of 
dynamic link flows at each node (14) combined with the FIFO condition on each link (17).

This problem does not necessarily have a feasible solution (satisfying constraints (24), (25) and (26)) due to the capacity constraint (25) on each link. The most extreme case for the nonexistence of a solution is when the time interval $I$ for the assignment is limited to an extremely short interval $\Delta t$. In this case, the OD travel demand $Q$ cannot be distributed into a time space and is forced to be distributed into only a network space (i.e., paths) while satisfying all the link capacity constraints. This implies that no feasible solution exists if $Q / \Delta t$, which can take an extremely large value, exceeds the maximum network capacity.

As shown later in Subsection 3.2, however, we can easily examine whether or not the problem [P-1] has a feasible solution for any network. Also, [P-1] always has feasible solutions (and an optimal solution) if the assignment time interval $I$ is large enough that we can make OD flow rates smaller than the maximum capacity of the underlying network. Thus, we concentrate our discussions on the relation between the optimal assignment and the equilibrium assignment. For the discussions in later sections, we note that any feasible solutions of [P-1] imply traffic flow patterns with no queuing congestion on the network.

Under this assumption, the most important property that characterizes the equilibrium assignment is that [P-1] is an equivalent optimization problem to the equilibrium conditions (14)(22). That is,

Proposition 1: For any networks with a single OD pair in which [P-1] has feasible solutions, the equilibrium assignment under the system of time-dependent tradable link permits minimizes the "social transportation cost" defined by (23).

\section{(Proof: see Appendix)}

On the surface, this proposition states that the equilibrium traffic patterns always coincide with the optimal traffic patterns for [P-1]. We should note, however, that problem [P-1] further provides information on the equilibrium prices/costs as well as the equilibrium flow patterns. Specifically, it is clear from the proof of Proposition 1 that $a$ ) the optimal Lagrange multipliers $\left\{\mathbf{p}^{*}(t)\right\}$ for constraint (25) coincide with the time-dependent link permit prices at equilibrium, $b$ ) the optimal Lagrange multiplier $\rho^{*}$ for constraint (24) gives the generalized transportation cost at equilibrium, and $c$ ) the optimal Lagrange multipliers $\left\{\pi^{*}(t)\right\}$ for (26) yield the transportation cost from the origin to each node at equilibrium.

In order to see more directly the fact that these Lagrange multipliers $\left(\rho^{*}, \pi^{*}(t), \mathbf{p}^{*}(t)\right)$ represent the equilibrium prices under the system of tradable permits, consider the dual problem, [D-1], of the optimal assignment problem [P-1]:

$$
\max _{(\boldsymbol{\rho}, \boldsymbol{\pi}, \mathbf{p}) \geq \mathbf{0}} F_{D}(\boldsymbol{\rho}, \boldsymbol{\pi}, \mathbf{p}) \equiv \rho Q-\sum_{i j \in L} \int_{0}^{T} p_{i j}(t) \mu_{i j} d t
$$

subject to

$$
\begin{array}{lr}
\rho \leq w(t)+\pi_{d}(t) & \forall t \in I \\
\pi_{j}\left(t+t_{i j}\right) \leq \pi_{i}(t)+\left(\alpha t_{i j}+p_{i j}(t)\right) & \forall t \in I, i j \in L
\end{array}
$$


From the duality theorem, the optimal value of the objective function of [D-1], $F_{D}{ }^{*}$, coincides with the optimal value of the objective function of $[\mathrm{P}-1], F_{P}{ }^{*}$, that is,

$$
\int_{0}^{T} q^{*}(t) w(t) d t+\alpha \sum_{i j \in L} \int_{0}^{T} y_{i j}^{*}(t) t_{i j} d t=\rho^{*} Q-\sum_{i j \in L} \int_{0}^{T} p_{i j}^{*}(t) \mu_{i j} d t .
$$

The right-hand side of this equation, $F_{P}{ }^{*}$, is the social transportation cost at the equilibrium under the system of tradable permits:

$$
\text { [total schedule cost] + [total travel time cost] }
$$

On the other hand, from the identity relation of transportation costs defined in Subsection 1.1, this should coincide with the value of

$$
\text { [total generalized transportation cost] - [total permits payments] }
$$

at equilibrium. Therefore, the right-hand side of (30), $F_{D}{ }^{*}$, should be the value of (31b) at equilibrium. Indeed, we can easily verify that ${F_{D}}_{D}{ }^{*}$ represents the social transportation cost at equilibrium with the form of (31b) if we interpret the optimal solution $\left(\rho^{*}, \pi^{*}(t), \mathbf{p}^{*}(t)\right)$ of [D-1] as the equilibrium prices under the tradable permits system. More specifically, the first term of $F_{D}{ }^{*}$ on the right-hand side of (30) denotes the equilibrium generalized transportation cost multiplied by the number of users, which is the total generalized transportation cost paid by users in the network. Note that this "cost" includes users' payments to the road manager to purchase permits, but these payments should not be counted as the "social cost" because they are just income transfers between the users and road manager. The total amount of these income transfers is given by the total market values of all the permits (i.e., the sum of the number of each permit $\mu_{i j}$ multiplied by its price $p_{i j}(t)$ for all links and times), which is given by the second term of $F_{D}{ }^{*}$. Thus, we see that $F_{D}{ }^{*}$ represents exactly the social transportation cost at the equilibrium with the form of (31b).

\subsection{Feasibility of the Assignment without Queuing}

We can examine whether or not the problem [P-1] has a feasible solution for any networks by simply constructing "time-space networks." The concept of “time-space network" may be best explained by the example of a train diagram. Denoting $x_{i}$ as the position of the $i$-th train in a 1dimensional guideway as a function of time $t$, we can depict the trajectories of trains, $\left\{x_{i}(t)\right\}$, in a 2dimensional (time, space) plane. For a set of appropriate time points $\left\{t_{k}\right\}$, the trajectories provide a set of points $\left\{\left(t_{k}, x_{i}\left(t_{k}\right)\right)\right\}$ in the plane; regarding these points as a set $N$ of "nodes," we can define a set $L$ of "links" by treating each segment of trajectories as a link connecting two nodes in $N$. The two sets $N$ and $L$ thus obtained constitute a time-space network $G(N, L)$ for the train diagram.

By applying a procedure similar to that for the train diagram above, we can construct the time-space network for analyzing dynamic flows in a general network. This is a powerful approach for solving a range of network problems with constant link travel times (for more detailed examples of this approach, see Ieda and Akamatsu (1988), Cascetta (2001)), although it is difficult to apply this to the problems with state-dependent link travel times. We can take advantage of this approach to check the feasibility of [P-1] since we do not need to consider queuing for the feasible solution of [P-1], which implies that the link travel times in [P-1] are constant.

Before describing the precise procedure for constructing the time-space network for [P-1], we first discretize time into small intervals of length $\Delta t$ : each time point is represented by $t=m \Delta t$, where $m=0,1,2, \ldots, \mathrm{M}$. We then assume that travel time $t_{i j}$ of each link is represented by a natural multiple of $\Delta t$ (i.e., an integer $n_{i j}$ satisfying $t_{i j}=n_{i j} \Delta t$ is given for each link). Under this setting, the 
“time-space network" is constructed by the following procedure:

Step 1-a. Replicates M copies of each node in the original network $G(N, L)$.

Step 1-b. For the expanded node set, add a single dummy node for the origin in $G$, and similarly, add a single new dummy node for the destination in $G$.

The set of nodes created by Step 1- $a$ and Step 1-b is the node set $N^{*}$ of the time-space network. To identify each node in $N^{*}$, we denote by $i(m)$ the node in $N^{*}$ that represents node $i$ at time $m$ $\Delta t$. We denote by $o^{*}$ the dummy node in $N^{*}$ for the origin $o$, and denote by $o^{*}$ in $N^{*}$ the dummy node for the destination $d$

Step 2-a. For every $m$, create a link going from node $i(m)$ toward node $j\left(m+n_{i j}\right)$ if there exists a link with travel time $t_{i j}=n_{i j} \Delta t$ in the original network $G(N, L)$. The attributes (i.e., travel cost and capacity) of each link created above is the same as those of the corresponding original link in $L$. Repeat this procedure for all links in $L$.

Step 2-b. For every $m$, create a link going from dummy node $o^{*}$ toward origin copy node $o(m)$ in $N^{*}$. For these new links, the travel cost is set to zero, and the capacity is infinity. For every time point $m$, create a link incident from destination copy node $d(m)$ toward dummy node $d^{*}$. For these new links, the travel cost is set to $w_{d}(m)$, and the capacity is infinity.

A set of links created by Step 2- $a$ and Step 2-b is the link set $L^{*}$ of the time-space network.

The dynamic system optimal assignment problem [P-1] (or its equivalent of the equilibrium problem (14)-(22)) reduces to a "static" minimum cost flow problem defined on the time-space network $G^{*}\left(N^{*}, L^{*}\right)$ constructed by the procedure above. More specifically, consider a static minimum cost flow problem defined on $G^{*}$, in which the origin node is given by the dummy node $o^{*}$, the destination node is the dummy node $d^{*}$, and the OD travel demand is given as $Q$. We then regard the flow on link $\left(i(m), j\left(m+n_{i j}\right)\right)$ in $G^{*}$ as the dynamic link flow rate $y_{i j}(m \Delta t)$ in the original network $G$, and the flow on link $\left(d(m), d^{*}\right)$ in $G^{*}$ as the dynamic OD flow rate $q(m \Delta t)$ in $G$. For this setting, the optimal solution of [P-1] is given by the link flow pattern in $G^{*}$ obtained as the solution of the minimum cost flow problem above.

To understand this fact, it is sufficient to check that all conditions of [P-1] are represented as the conditions for the minimum cost flow problem defined on $G^{*}$. We first see that conservation of the dynamic flow in [P-1] is automatically represented by conservation of the static flow at each node on $G^{*}$. Constraint (26) is satisfied by the flow conservation at each node $i(m)$ on $G^{*}$; constraint (24) is satisfied by the flow conservation at each dummy node $d$ in Step 2-a of the procedure to construct $G^{*}$. We also see that the capacity constraint of each link (25) for each time period corresponds to the capacity constraint of each link on $G^{*}$. Furthermore, the objective function of [P$1]$ is consistently represented by the total travel cost in the minimum cost flow problem defined for $G^{*}$ : the first term of the objective function is represented by the sum of travel costs for the set of links $\left(d(m), d^{*}\right)$ constructed in Step 2-b, and the second term is the sum of travel costs for the set of links constructed in Step 2-a.

Thus, the dynamic optimal assignment problem [P-1] is reduced to solving a static minimum cost flow problem on $G^{*}$. The static minimum cost flow problem has been well studied in computer science (see, for example, Kennington and Helgason (1980), Ahuja, Magnanti and Orlin 
(1993)), and very efficient (polynomial order) algorithms are available for solving this problem. Therefore, we see that the feasibility of [P-1] can be easily verified by using these algorithms.

\section{Congestion Pricing vs. Tradable Permits}

\subsection{Perfect Information Case}

The equilibrium permit prices formulated in Section 2 can be interpreted as the optimal toll levels for a congestion pricing scheme. To see this, consider a "dynamic congestion pricing scheme" in which the road manager imposes a time-dependent toll (congestion tax) for each link in the network. Denoting by $p_{i j}(t)$ the toll of link $(i, j)$ for a user arriving at the link at time $t$, we easily see that all transportation costs required for a user to make a trip have precisely the same form as the transportation costs under the permit system in Section 2. Consequently, it is obvious that the equilibrium states for the dynamic congestion pricing with a toll pattern $\{\mathbf{p}(t)\}$ satisfy the conditions (14)-(21). In other words, we can interpret the conditions (14)-(21) as those for defining the equilibrium flow pattern $\{\mathbf{y}(t)\}$ that arises when the road manager sets a dynamic toll pattern $\{\mathbf{p}(t)\}$. With this in mind, suppose that the road manager wishes to prevent the occurrence of queuing congestion in the network by setting an appropriate toll pattern $\left\{\mathbf{p}^{*}(t)\right\}$ such that the equilibrium inflow rate $y_{i j}(t)$ of each link never exceeds the capacity $\mu_{i j}$. (i.e., queuing congestion never occurs). Then, it follows that the conditions for achieving this goal are given by the same form as (22). That is, condition (22), which represents the market clearing (demand-supply equilibrium) condition for the tradable permit scheme, is now the condition for the road manager to achieve the optimal flow pattern under the congestion pricing scheme. Thus, the system of equations (14)-(22) can be viewed as the conditions for obtaining the optimal toll pattern when the equilibrium flow pattern does not cause queuing congestion.

As we have seen in Proposition 1, the system of equations (14)-(22) are also equivalent to the optimal assignment problem [P-1], and therefore, the pair $\left\{\mathbf{p}^{*}(t), \mathbf{y}^{*}(t)\right\}$ of the optimal toll pattern and the resulting equilibrium flow pattern exist if [P-1] has feasible solutions. Therefore, we obtain the following proposition on the equivalence of the tradable permit scheme and the congestion pricing scheme:

Proposition 2: Consider any networks with a single $O D$ pair in which [P-1] has feasible solutions, and further suppose that the road manager has perfect information about the transportation demands. Then, the equilibrium assignment under the system of time-dependent tradable link permits coincides with the equilibrium assignment under the system of optimal dynamic congestion pricing.

\subsection{Imperfect Information Cases: Economic Losses due to Congestion Pricing}

The proposition above states that a congestion pricing scheme under a certain condition attains the same ideal state as does the tradable permit scheme. However, we should pay attention to the fact that the required conditions for attaining the ideal state in the tradable permit scheme are largely different from those in the congestion pricing scheme. The differences lie in the amount and accuracy of the information needed for the authority (i.e., the road manager) to implement the regulation. In the tradable permit scheme, what the road manager should know is just the traffic capacity of each link (and queuing congestion can be eliminated just by issuing the number of permits for each link that is equal to or less than the link capacity). In the congestion pricing scheme, 
on the other hand, it is not just traffic capacity that the road manager is required to know; it is obvious from the discussion above that the road manager cannot calculate an appropriate toll pattern that prevents congestion without having accurate information on the users' behaviors (i.e., precise demands).

Considering the differences between the two schemes, the desirable transportation demand management scheme must be found. In generalized terms, this becomes the problem of comparing between "quantity-based regulation" and "price-based regulation." In the field of economics, there have been many studies concerning the comparison of the two regulation schemes. According to the standard theory (see, for example, Weizman (1974), Laffont (1977)), quantity-based regulation produces more efficient outcomes than price-based regulation if a regulation authority has only imperfect information on the demand side conditions (i.e., demand functions) while having perfect information on the supply side conditions (i.e., supply functions). For the problem in this paper, we obtain a similar conclusion, although the underlying assumptions of our problem are different from those in conventional economic theory.

We show this fact below in more concrete terms. In our problem, what corresponds to "supply side conditions" is the network performance (traffic capacity of each link), for which information can be obtained accurately with relatively small effort. On the other hand, the information on the "demand side conditions" is the OD demand $Q$, the schedule cost function $w(t)$, the travel time pattern $\boldsymbol{t}$ and the value of time $\alpha$. It is not easy for the road manager to obtain this information accurately.

It is therefore natural to think of the events that happen in the dynamic congestion pricing scheme if the demand side information is not perfect. As a simple example, suppose that the road manager estimates the users' value of time as $\beta$, which is different from the true value of time $\alpha$. Under this assumption, the road manager solves (14)-(22), and then obtains a toll pattern $\left\{\mathbf{p}^{\prime}(t)\right\}$ that is different from the true optimal toll pattern $\left\{\mathbf{p}^{*}(t)\right\}$. It is obvious that the traffic pattern $\left\{\mathbf{y}^{\prime}(t)\right\}$ arising under the incorrect toll pattern $\left\{\mathbf{p}^{\prime}(t)\right\}$ does not minimize the objective function of [P-1] (i.e., the sum of the schedule cost and travel cost), which causes economic loss in the social transportation cost. Worse still, there is no guarantee that the capacity constraint in each link is satisfied in the traffic flow patterns $\left\{\mathbf{y}^{\prime}(t)\right\}$; that is, queuing congestion may occur in the network. The reason is as follows: the manager believes that a traffic flow pattern $\left\{\mathbf{y}^{\prime \prime}(t)\right\}$ should arise assuming that the users' value of time is $\beta$ and the toll pattern is $\left\{\mathbf{p}^{\prime}(t)\right\}$, which is determined such that $\left\{\mathbf{y}^{\prime} '(t)\right\}$ satisfies capacity constraint (22); but the flow pattern arising in reality is $\left\{\mathbf{y}^{\prime}(t)\right\}$ (i.e., the flow pattern that arises when the users' value of time is $\alpha$ and the toll pattern is $\left.\left\{\mathbf{p}^{\prime}(t)\right\}\right)$, and it follows that there is no necessity that $\left\{\mathbf{y}^{\prime}(t)\right\}$ satisfies (22). Note here that the social transportation cost in the optimal assignment problem [P-1] is defined under the assumption that queuing congestion never occurs. Consequently, in the congestion pricing scheme, any incorrect toll pattern $\left\{\mathbf{p}^{\prime}(t)\right\}$ not only minimizes the sum of the schedule cost and travel cost but also causes additional economic loss due to the occurrence of queuing congestion.

On the other hand, the tradable permit system never produces the above economic loss in theory, if the road manager just has correct information on the link capacities. Of course, there may be the possibility in reality that appropriate (theoretically correct) permit prices do not occur in the tradable permits market. This corresponds to the possibility of setting the incorrect toll pattern $\left\{\mathbf{p}^{\prime}(t)\right\}$ in the congestion pricing scheme. It may be said from conventional wisdom that the degree 
of mispricing in the markets is likely to be lower than that of the authority's mispricing. Furthermore, even if the degrees of mispricing are at a comparable level, there is a significant difference in the resulting effects of "distorted prices" between the two schemes. The effect of the distorted prices in the tradable permit system is confined to the loss in only the permit trading markets. They are irrelevant to the occurrence of queuing in the network, unlike the congestion pricing scheme. This is because the definition of the permit system ensures that the traffic flow never exceeds the number of permits issued, no matter how "incorrect" the permit prices are; thus, the tradable permit system never produces additional economic losses due to the occurrence of queuing, as observed in the congestion pricing system.

Still, one might argue that there may be the possibility in reality that queuing congestion occurs in the tradable permit system if the users' arrival times at each link are not accurate. However, the possibility that such a phenomenon occurs as well as the resulting losses are all the same in dynamic congestion pricing, too. Hence, this can be ignored from the standpoint of the relative comparison of these two schemes. Thus, the discussion above can be summarized by the following Proposition:

Proposition 3: Consider any networks with a single OD pair in which [P-1] has feasible solutions, and further suppose that the road manager does not have perfect information about the transportation demands. Then, the equilibrium assignment under a system of dynamic congestion pricing incurs a larger total transportation cost than that in the equilibrium under a system of time-dependent tradable link permits.

\section{Self-financing Principle}

In order for the "market selling scheme" of the tradabale network permits to be socially acceptable, it may be desirable to redistribute the revenue from selling the permits to the road users. As a benchmark scheme of the redistribution, it is significant to consider the case in which the revenue is used for financing the capacity expansion of the network. For this type of redistribution scheme in congenstion pricing, the "self-financing principle" has been well known since the pioneering work of Mohring and Harwitz (1962): for a congestible facility with a constant long-run average cost, the revenue from the optimal congestion pricing exactly covers the cost of the optimal capacity. A considerable number of studies have been made on whether or not this principle holds for a variety of assumptions (see, for example, Keeler and Small (1977), Arnott and Kraus (1995), Arnott and Kraus (1998), Yang and Meng (2002)). Accordingly, it is worthwhile to investigate whether this principle applies to the equilibrium revenue of tradable permits. As the first step of this investigation, Akamatsu (2007a) showed that the principle also applies for the tradable permit scheme for a single bottleneck. As we shall see below, this principle can be extended to the scheme of general networks.

Consider the following minimization problem [P-K]:

$$
\begin{aligned}
& \min _{(\mathbf{q}, \mathbf{y} \cdot \mu) \geq \mathbf{0}} K(\mu)+F_{P}(\mathbf{q}, \mathbf{y}) \\
& \text { subject to (24), (25) and (26). }
\end{aligned}
$$

This problem is almost the same as [P-1] except that $a$ ) the unknown (control) variables in [P-K] are 
not only the flow pattern $(\mathbf{q}, \mathbf{y})$ but also the link capacity pattern $\mu$ that is assumed to be constant in [P-1], b) the investment cost $K$ as a function of $\mu$ is added into the objective function $F_{p}$ of [P-1], which means that the social cost we would like to minimize is defined as the sum of the total transportation cost and the investment costs required for the increases in network capacity. The investment cost function $K$ is assumed to be homogeneous of degree 1 with respect to $\mu$; that is,

$$
K(\mu)=\sum_{i j \in L} \frac{\partial K(\mu)}{\partial \mu_{i j}} \mu_{i j}
$$

The optimality conditions of [P-K] are similar to those of [P-1]. The only difference is in the addition of the following condition for the optimality of link capacity $\mu$ :

$$
\mu_{i j}^{*} \frac{\partial L}{\partial \mu_{i j}}=0, \frac{\partial L}{\partial \mu_{i j}} \geq 0, \mu_{i j}^{*} \geq 0, \quad \forall i j \in L
$$

where $L$ is the Lagrangean function of $[\mathrm{P}-\mathrm{K}]$, and the derivative with respect to $\mu$ is given as

$$
\frac{\partial L}{\partial \mu_{i j}}=\frac{\partial K(\mu)}{\partial \mu_{i j}}-\int_{0}^{T} p_{i j}(t) d t
$$

Substituting (35) into (34), and summing the first equation of (34) for all links, we obtain

$$
K\left(\mu^{*}\right)=\sum_{i j \in L} \mu_{i j}^{*} \int_{0}^{T} p_{i j}^{*}(t) d t,
$$

where we use the homogeneity property (33) of function $K$. The left-hand side of (36) is the investment cost required for achieving the optimal link capacity pattern. The right-hand side of (36) is the total market value of the link permits at equilibrium under the tradable network permit system; this can be seen from the fact that, for any capacity pattern, the optimal Lagrange multiplier $\left\{\mathbf{p}^{*}(t)\right\}$ of [P-1] coincides with the equilibrium permit prices, as shown in Section 3. Therefore, (36) means that the "self-financing principle" holds for the tradable network permit scheme. This can be summarized as follows:

Proposition 4: Consider any networks with a single OD pair in which [P-1] has feasible solutions, and further suppose that the investment cost function $K(\mu)$ is homogeneous of degree 1 in link capacities $\mu$. Let the optimal investment cost be the cost required for increasing the link capacities so as to minimize the social cost defined in (32). Then the optimal investment cost is equal to the total market value of bottleneck permits (i.e., total revenue of the road manager); that is, the "self-financing principle" holds for the tradable network permits system.

\section{Concluding Remarks}

This paper considered a system of bottleneck tradable permits for general networks (“tradable network permits") with a single OD pair. We first provided a model that describes timedependent flow patterns at equilibrium under the proposed system. We then revealed that the equilibrium coincides with the optimal assignment pattern that minimizes the social transportation cost (i.e., the equilibrium under the proposed system is efficient). We further showed the theoretical relationship between the tradable permit system and congestion pricing: we 
demonstrated the definite advantages of the tradable permit system over the congestion pricing system when the demand information is not perfect, whereas they are equivalent for the perfect information case. Finally, we proved that the self-financing principle holds in the proposed scheme (i.e., the total market value of the permits is equal to the investment cost required for increasing the network capacity up to a socially optimal level).

We showed the efficiency of the tradable network permits system from the viewpoint of aggregated social costs (i.e., minimization of total transportation cost). This result, however, does not necessarily mean that the introduction of the proposed system always leads to Pareto improvement. In a single bottleneck case, the Pareto improvement can be proved since there is a one-to-one correspondence between the queuing delay (at equilibrium without the system) and the permit price (at equilibrium with the system). For networks with many bottlenecks, there are complex interactions among queuing delays at equilibrium without the system (see, for example, Kuwahara and Akamatsu (1993), Akamatsu (2001), Akamatsu and Heydecker (2003)); the correspondences between the equilibrium queuing delays and the permit prices are not straightforward. Futher exploration on the Pareto improvement property of tradable network permits is one of the important issues that should be addressed in future research.

The results of this paper can be extended in various directions. For instance, it is straightforward to generalize the model to allow for many-to-many OD patterns. In that case, we again find that the equilibrium is efficient in the sense that the total transportation cost in the network is minimized. Another straightforward extension is to consider elastic OD demands. It can be shown that the efficiency of the tradable network permit system applies to this case, too. In addition to the extensions on the "demand side," we can also generalize the theory to include "supply side" conditions: the equilibrium permit prices can be exploited for obtaining dynamic capacity allocation policies (e.g., signal control policies) that minimize the total transportation cost. More discussion of these issues can be found in Akamatsu (2007b).

Acknowledgement: The research was partially supported by the Japan Society for the Promotion of Science, Grant-in-Aid for Exploratory Research 18656142. 


\section{Appendix}

\section{Proof of Proposition 1:}

We will show that a necessary and sufficient condition for the optimality of the optimization problem [P-1] coincides with the equilibrium conditions (14)-(22). To derive the optimality conditions, we first define Lagrangean function $L$ for the problem [P-1]:

$$
\begin{aligned}
L(\mathbf{q}, \mathbf{y}, \boldsymbol{\rho}, \boldsymbol{\pi}, \mathbf{p}) & \equiv F_{P}(\mathbf{q}, \mathbf{y}) \\
& +\rho\left\{Q-\int_{0}^{T} q(t) d t\right\}+\sum_{i j \in L} \int_{0}^{T} p_{i j}(t)\left\{y_{i j}(t)-\mu_{i j}\right\} d t \\
& +\sum_{i \in N} \int_{0}^{T} \pi_{i}(t)\left\{q(t) \delta_{i d}+\sum_{k} y_{i k}(t)-\sum_{k} y_{k i}\left(t-t_{k i}\right)\right\} d t,
\end{aligned}
$$

where function $F_{P}$ is the objective function of [P-1] defined in (23); $\rho,\{\mathbf{p}(t)\}$, and $\{\pi(t)\}$ are Lagrange multipliers corresponding to constraints (24), (25), and (26), respectively. Then, the necessary and sufficient conditions for the optimality of [P-1] are given by the following KuhnTucker conditions:

$$
\begin{aligned}
& \left\{\begin{array}{l}
\partial L / \partial q^{*}(t)=0 \text { if } q^{*}(t)>0 \\
\partial L / \partial q^{*}(t) \geq 0 \text { if } q^{*}(t)=0
\end{array}\right. \\
& \left\{\begin{array}{l}
\partial L / \partial y_{i j}^{*}(t)=0 \text { if } y_{i j}^{*}(t)>0 \\
\partial L / \partial y_{i j}^{*}(t) \geq 0 \text { if } y_{i j}^{*}(t)=0
\end{array}\right. \\
& \partial L / \partial \rho^{*}=0 \\
& \left\{\begin{array}{l}
\partial L / \partial p_{i j}^{*}(t)=0 \text { if } p_{i j}^{*}(t)>0 \\
\partial L / \partial p_{i j}^{*}(t) \leq 0 \text { if } p_{i j}^{*}(t)=0
\end{array}\right. \\
& \partial L / \partial \pi_{i}^{*}(t)=0 \\
& \forall t \in I, \forall i j \in L \\
& \forall t \in I, \forall i j \in L \\
& \forall t \in I, \forall i \in N
\end{aligned}
$$

It can be easily seen that conditions (A.4), (A.5), and (A.6) reduce to the equilibrium conditions (20), (22), and (14), respectively. To examine conditions (A2) and (A.3), we calculate the partial derivatives of the Lagrangean function:

$$
\begin{aligned}
& \partial L / \partial q^{*}(t)=w(t)+\pi_{d}(t)-\rho \\
& \partial L / \partial y_{i j}^{*}(t)=\left(\alpha t_{i j}+p_{i j}(t)\right)+\pi_{i}(t)-\pi_{j}\left(t+t_{i j}\right)
\end{aligned}
$$

Substituting (A.7) into (A2), we have the same form of conditions as in equilibrium condition (21); similarly, we see that (A2) reduces to the equilibrium condition (18). Thus, Lagrange multipliers $\mathbf{p}^{*}(t), \pi^{*}(t)$, and $\rho^{*}$ in the optimality conditions (A.2)-(A.6) coincide with the equilibrium link permit prices, equilibrium minimum path costs, and the equilibrium generalized transportation costs in equilibrium conditions (14)-(22); the optimal flow patterns $\left(\mathbf{q}^{*}(t), \mathbf{y}^{*}(t)\right)$ also coincide with the equilibrium flow patterns. Q.E.D. 


\section{References}

Ahuja,R.K., T.L. Magnanti, J.B. Orlin. 1993. Network flows: Theory, algorithms and applications. Prentice-Hall.

Akahane,H., M. Kuwahara. 1996. A basic study on trip reservation systems for recreational trips on motorways. In: Proc. of the $3^{\text {rd }}$ World Congress on Intelligent Transportation Systems 1-7, ITS America, Washington D.C.

Akamatsu, T. 2001. An efficient algorithm for dynamic traffic equilibrium assignment with queues. Transportation Science 35, 389-404.

Akamatsu, T. 2007a. Tradable bottleneck permits: Optimal allocation of priority road services submitted to Journal of Urban Economics.

Akamatsu, T. 2007b. Distributed signal control and traffic assignment through tradable network permits submitted to Transportation Research part $B$.

Akamatsu, T., B. Heydecker. 2003. Detecting capacity paradoxes in dynamic traffic assignment in saturated networks. Transportation Science 37, 123-138.

Akamatsu, T., M. Kuwahara 1994. Dynamic user equilibrium assignment on over-saturated road networks for a one-to-many/many-to-one OD pattern. JSCE Journal of Infrastructure Planning and Management IV-23, 21-30.

Akamatsu, T., S. Sato, X.L. Nguyen. 2006. Tradable time-of-day bottleneck permits for morning commuters. JSCE Journal of Infrastructure Planning and Management 62, 605-620.

Arnott, R., M. Kraus. 1995. Financing capacity in the bottleneck model. Journal of Urban Economics 38, 272-290.

Arnott, R., M. Kraus. 1998. Self-financing of congestible facilities in a growing economy. In: Topics in Public Economics, (eds.) Pines,D. et al., 161-184.

Arnott, R., A. de Palma, R. Lindsey. 1993. A structural model of peak-period congestion: A traffic bottleneck with elastic demand. American Economic Review 83, 161-179.

Cascetta, E. 2001. Transportation systems engineering: Theory and methods. Kluwer Academic.

Daganzo, C.F. 1985. The uniqueness of a time-dependent equilibrium distribution of arrivals at a single bottleneck. Transportation Science 19, 29-37.

Fujita, M. 1989. Urban economic theory, Cambridge University press.

Ieda, H., T. Akamatsu, J. Takagi. 1988. Time-space network equilibrium model of rail commuters and its application to the evaluation of train diagrams from the view point of user welfare. Infrastructure Planning Review 6, 177-184.

Keeler, T.E., K.A. Small. 1977. Optimal peak-load pricing, investment and service levels on urban expressways. Journal of Political Economy 85, 1-25.

Kennington, J.L., R.V. Helgason. 1980. Algorithms for network programming. Wiley-Interscience.

Kuwahara, M. 1990. Equilibrium queuing patterns at a two-tandem bottleneck during the morning peak. Transportation Science 24, 217-229.

Kuwahara, M., T. Akamatsu. 1993. Dynamic equilibrium assignment with queues for a one-tomany OD pattern. Transportation and Traffic Theory 12, 185-204.

Laffont, J.J. 1977. More on prices vs. quantities. 1977. The Review of Economic Studies 44, 177182.

Mohring, H., M. Harwitz. 1962. Highway benefits: An analytical framework. Northwestern University Press.

Smith, M.J. 1984. The existence of a time-dependent equilibrium distribution of arrivals at a single bottleneck. Transportation Science 18, 385-394.

Teodorovic, D., P. Edara. 2005. Highway space inventory control system. Transportation and 
Traffic Theory 16, 43-62.

Verhoef, E., P. Nijkamp, P. Rietveld. 1997. Tradable permits: Their potential in the regulation of road transport externalities. Environment and Planning 24B, 527-548.

Vickrey, W.S. 1969. Congestion theory and transportation investment. American Economic Review 59, 251 -260.

Weitzman, M. L. 1974. Prices vs. quantities. The Review of Economic Studies 41 477-491.

Wong, J-T. 1997. Basic concepts for a system for advance booking for highway use. Transport Policy 4, 109-114.

Yang, H., Q. Meng. 2002. A note on "highway pricing and capacity choice in a road network under build-operate-transfer scheme”. Transportation Research 36A, 659-663.

(Revised March 3, 2007) 